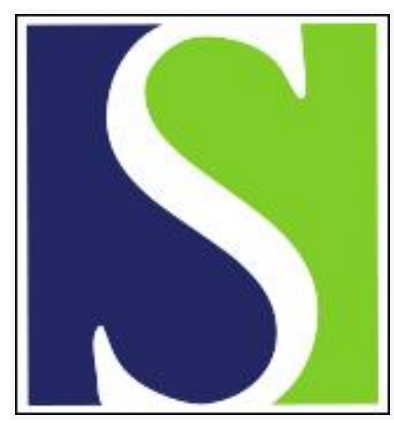

Scand J Work Environ Health 2013;39(3):259-267

https://doi.org/10.5271/sjweh.3328

Published online: 11 Oct 2012, Issue date: 01 May 2013

Relative weight and disability retirement: a prospective cohort study

by Roos E, Laaksonen M, Rahkonen O, Lahelma E, Lallukka T

Severely obese and obese employees have a clearly elevated risk for disability retirement, especially for musculoskeletal causes. The adverse effects of obesity on work ability are partly dependent on co-morbidities and ensuing lowered physical functioning. Promoting the maintenance of normal weight among employees is important as well as the effective treatment of obesity and its co-morbidities.

Affiliation: Hjelt Institute, Department of Public Health, PO Box 41, 00014 University of Helsinki, Finland. eira.roos@fimnet.fi

Refers to the following text of the Journal: 2006;32(1):5-11

The following articles refer to this text: 2013;39(3):233-240; 2013;39(3):217-220; 2013;39(3):241-258; 2017;43(5):426-435; 2019;45(4):376-385

Key terms: BMl; body mass index; disability retirement; Finland; obesity; occupational health; occupational health; prospective cohort study; relative weight; sick leave; weight; work ability; work disability

This article in PubMed: www.ncbi.nlm.nih.gov/pubmed/23060294 


\title{
Relative weight and disability retirement: a prospective cohort study
}

\author{
by Eira Roos, MD, ${ }^{1}$ Mikko Laaksonen, PhD, ${ }^{1}$ Ossi Rahkonen, PhD, ${ }^{1}$ Eero Lahelma, PhD, ${ }^{1}$ Tea Lallukka PhD ${ }^{1}$
}

\begin{abstract}
Roos E, Laaksonen M, Rahkonen 0, Lahelma E, Lallukka T. Relative weight and disability retirement: a prospective cohort study. Scand J Work Environ Health. 2013;39(3):259-267. doi:10.5271/sjweh.3328

Objectives The aims of this study were to examine (i) the association of relative weight with subsequent disability retirement due to any diagnosis and also in two major diagnostic groups (ie, musculoskeletal diseases and mental disorders) and (ii) whether diagnosed diseases, physical and mental functioning, and working conditions explain these associations.

Methods This prospective study comprised a cohort of 6542 middle-aged employees of the City of Helsinki, Finland. Questionnaire data were linked with register data on disability retirements, with a mean follow-up time of 7.8 years.

Results Adjusting for age, body mass index (BMI) was associated with all-cause disability retirement among men and women, the highest risk being for the severely obese and the obese [hazard ratio (HR) 3.45, 95\% confidence interval (95\% CI) 2.53-4.69; HR 1.94, 95\% CI 1.52-2.46, respectively]. Adjusting for age, relative weight was also strongly associated with the main retirement diagnoses, especially musculoskeletal diseases among the severely obese (HR 4.76, 95\% CI 3.06-7.40) and obese (HR 2.35, 95\% CI 1.62-3.39). The association was attenuated when adjusting for self-reported diseases and physical and mental functioning at baseline. Working conditions had negligible effects on the associations.
\end{abstract}

Conclusions Maintenance of normal weight is likely to reduce the risk of disability retirement. Among obese employees, the focus should be on the improvement of physical functioning and the effective treatment of obesity and its co-morbidities to counteract the heightened risk of disability retirement.

Key terms BMI; body mass index; Finland; obesity; occupational health; sick leave; weight; work ability; work disability.

Obesity is a prevalent and serious public health problem that is associated with numerous chronic diseases (1) and increased mortality (2). Obesity also affects individual well-being (3) and quality of life (4). Among employees, obesity is associated with increased risk and duration of sick leave $(5,6)$ and workplace injuries $(7)$. There is also evidence that obesity is associated with employees' decreased functioning and productivity at work (8). As obesity is a common condition among middle-aged employees, it creates a major economic burden in workplaces and on society (9).

The work-related and economic consequences of obesity are further accentuated by obesity-associated premature retirement $(10,11)$. In addition to obesity, being under- or overweight may also contribute to the risk of disability retirement as relative weight measured by body mass index (BMI) tends to have a J-shaped association with disability retirement (11). However, the evidence of the association between underweight and disability retirement is not conclusive, and has been mainly shown for men (11).

Few previous studies have included data on diagnoses leading to disability retirement (12-14). A Finnish study found that obese men and women had an elevated risk of disability retirement due to cardiovascular and musculoskeletal diseases but not due to mental disorders (12). Two Swedish studies analyzed only men. In the first study (13), the obese were at increased risk of disability retirement due to musculoskeletal and cardiovascular diseases and mental disorders. Among the underweight, alcohol dependence as a cause for retirement was more common than among those with

1 Hjelt Institute, Department of Public Health, University of Helsinki, Helsinki, Finland.

Correspondence to: Eira Roos, Hjelt Institute, Department of Public Health, PO Box 41, 00014 University of Helsinki, Finland. [E-mail: eira.roos@fimnet.fi] 
higher BMI. In the second study (14), the risk for disability retirement due to mental disorders was similarly increased for the under- and overweight compared to normal-weight men. The risk for disability retirement due to cardiovascular and musculoskeletal diseases was increased among the overweight and obese but not among underweight men.

According to the Finnish retirement legislation, an employee has to have a diagnosed illness before a disability pension can be granted. However, diagnosed illness is not enough to induce disability retirement; the employee also has to have lowered work ability due to the illness, either mentally or physically, to qualify for disability retirement. In addition to illnesses and functioning, working conditions are considered when work ability is evaluated. For example, physical health problems may lower one's work ability more when doing physically demanding work compared to, for example, desk or office work. In earlier studies, health status and working conditions have only rarely been taken into account. A previous Swedish study adjusted the association between BMI and disability retirement for hypertension, which slightly attenuated the association (13), but other diseases were not included. Another Swedish study (14) found that, after adjusting for muscular strength, the risk for disability retirement was even higher among the obese but lower among the underweight. No other measures of health status have been considered. Working conditions have only rarely been included, despite their potential importance for work disability (15). A Danish study of nurses (16) found that both BMI and working conditions, such as worktime arrangements and physical demands of the work, were associated with disability retirement, but their combined effect was not reported. In order to understand the associations between weight and disability retirement, it is vital to take into account key covariates including health, functioning, and working conditions.

The aim of our study was first to examine the association of relative weight with subsequent disability retirement due to any cause, as well as due to musculoskeletal diseases, mental disorders, and other causes. Our second aim was to examine whether health status, physical and mental functioning, and working conditions explain these associations.

\section{Methods}

Data

The study was based on the Helsinki Health Study cohort. The baseline data were derived from mail questionnaire surveys among the employees of the City of Helsinki, Finland. The baseline surveys were conducted in 2000, 2001, and 2002 among employees who reached $40,45,50,55$, or 60 years during each year $(\mathrm{N}=8960$, response rate $67 \%$ ) (17). According to non-response analysis, the baseline data represents the target population satisfactorily $(17,18)$. There were 5131 women and 1411 men in the study population. By socioeconomic position, $31 \%$ of the respondents were classified as managers and professionals, $19 \%$ as semi-professionals, $36 \%$ as routine non-manual employees, and $14 \%$ as manual workers. The respondents represent municipal occupations, such as nurses, teachers, social workers, fire-fighters, bus drivers, and office workers.

Height and weight were taken from the baseline surveys. BMI $\left(\mathrm{kg} / \mathrm{m}^{2}\right)$ was calculated from self-reported data and categorized as: $<20$ (underweight), 20-24.9 (normal weight), 25-29.9 (overweight), 30-34.9 (obese), and $\geq 35$ (severely obese). Participants with missing data on weight or height $(\mathrm{N}=49)$ and pregnant women $(\mathrm{N}=15)$ were excluded.

Disability retirement data were derived from the Finnish Centre for Pensions national register data on all retirement events. These data were linked to the baseline survey data using unique personal identification numbers. The data linkage was done among respondents who gave their written consent for such linkage $(74 \%)$. Non-consenter analyses have been reported elsewhere $(17,18)$. These showed that consenters represented the target population satisfactorily. Disability retirement was followed up until the end of 2010. Participants who retired due to their age or died before disability retirement were censored. Participants who turned 63 years were also censored as in Finland disability pension cannot be granted after the age of 63 years. The mean follow-up time was 7.8 years.

There were 681 disability retirement events during the follow-up time. The disability retirement data include complete information on the main diagnosis (according to ICD-10, International Classification of Diseases, Tenth Revision) (19) of the retirement. The diagnoses were grouped into musculoskeletal diseases (diseases of the musculoskeletal system and connective tissue, ICD-10 codes M00-M99), mental disorders (mental and behavioral disorders, ICD-10 codes F00-F99), and other causes. Of all the disability retirees, 290 retired due to musculoskeletal diseases, 198 due to other causes, and 193 due to mental disorders.

The ethics committees at the Department of Public Health, University of Helsinki, and the City of Helsinki Health Authorities approved the study.

\section{Covariates}

Covariates taken from the baseline included diagnosed diseases, physical and mental functioning, and working conditions. First, diseases were assessed by a question 
asking whether the respondent had ever been diagnosed with any of the listed diseases, which included musculoskeletal diseases (gout, arthrosis, osteoarthritis), cardiovascular diseases (angina pectoris, myocardial infarction, cerebrovascular stroke, claudication), mental disorders (depression, anxiety, other mental health problems), diabetes, cancer, and eating disorders. If the respondent reported at least one such disease, a health problem was present.

Second, physical and mental health functioning were measured using the physical and mental component summaries of the Short-Form 36 (SF-36) health questionnaire (20). Summary scores of both physical and mental component scores were divided into quartiles.

Third, working conditions included shift work, physical working conditions, and psychosocial working conditions. Shift work was encoded as a dichotomous covariate: employees doing regular daytime work formed one group, and the rest, including those doing any type of shift or nighttime work, formed another group. Factor analysis of an 18-item inventory of physical working conditions developed at the Finnish Institute of Occupational Health (21) yielded the following factors: (i) physical workload, such as uncomfortable postures, repetitive trunk rotation, repetitive movements, and heavy physical exertion; (ii) hazardous exposures, such as dirt, dampness, weak lightning, solvents, gases or irritating substances, heat, cold, draft, mold, noise, and vibration; and (iii) computer work, including sedentary work, using a computer mouse, and working with a computer display terminal. Each factor score was divided into quartiles. Karasek's job demand job control questionnaire was used to measure psychosocial working factors (22). Separate summary scores for job demands (9 items) and job control (9 items) were divided into quartiles

\section{Statistical analysis}

First, the incidence of different disability retirement events across the BMI groups was calculated. The rates are reported per 100 person-years. Next, Cox regression analysis was used to examine the associations between BMI and all subsequent disability retirement among women and men separately.

In the analyses, five different models were fitted. Model 1 was adjusted for age. Model 2 was adjusted for age and diseases. Model 3 was adjusted for age and physical and mental health functioning. Model 4 was adjusted for age and all the working conditions at the same time. Model 5 was adjusted for all covariates of the previous models simultaneously. Socioeconomic position, smoking, and physical activity were adjusted for in sensitivity analyses, but as their contributions to the examined associations were negligible, they were omitted from the final models. As the number of men was rather small in our sample, we tested the interaction between gender and disability retirement, and when no interaction was found, the data for women and men were pooled for the diagnosis-specific analyses. When analyzing data on men and women together, all models were adjusted additionally for gender. The results are presented as hazard ratios (HR) and their $95 \%$ confidence intervals (95\% CI).

\section{Results}

At baseline, $32 \%$ of women and $46 \%$ of men were overweight and $15 \%$ of both women and men were obese (table 1). Disability retirement was more common in the higher BMI groups. Severely obese men and women had a four-time greater incidence of all-cause retirement than normal-weight men and women. When examining the disability retirement diagnoses, the incidence of disability retirement due to musculoskeletal diseases was 5.6-fold greater among severely obese than normal-weight women, and 11-fold greater among the severely obese than normal-weight men. The incidence of disability retirement due to mental disorders was not increased among severely obese men, but severely obese women had 2.7-fold greater incidence than women of normal weight.

Obese employees were somewhat older and had more diagnosed diseases and lower physical functioning compared to normal-weight employees (table 2). Among women, shift work, physical workload, and hazardous exposures at work were more common among the obese, and computer work was less common than among normal-weight employees. Among men, hazardous exposures were more common among the underweight than in other BMI groups and physical workload was less among normal-weight men than in other BMI groups.

\section{All-cause retirement}

After adjusting for age, severely obese (HR 3.47, 95\% CI 2.47-4.87), obese (HR 2.05, 95\% CI 1.58-2.67), and overweight women (HR 1.38, 95\% CI 1.11-1.72) had a higher risk of disability retirement (table 3). Being underweight was not statistically significantly associated with disability retirement (HR $1.30,95 \%$ CI $0.82-1.93$ ).

After adjusting for diagnosed diseases, the association between BMI and all-cause retirement attenuated, but the elevated risk remained among the severely obese and obese. After adjusting for physical and mental functioning, the association between BMI and disability retirement strongly attenuated, but among the obese and 
Table 1. Incidence of all-cause disability retirement and diagnosis-specific disability retirement per 100 person-years by gender and body mass index (BMI).

\begin{tabular}{|c|c|c|c|c|c|c|c|}
\hline \multirow[t]{2}{*}{$\mathrm{BMI}\left(\mathrm{kg} / \mathrm{m}^{2}\right)$} & \multirow[t]{2}{*}{$\mathrm{N}$} & \multirow[t]{2}{*}{$\%$} & \multirow[t]{2}{*}{ Person-years } & \multicolumn{4}{|c|}{ Incidence of disability retirement per 100 person-years } \\
\hline & & & & $\begin{array}{l}\text { All-cause disability } \\
\text { retirement }\end{array}$ & $\begin{array}{c}\text { Musculoskeletal } \\
\text { diseases }\end{array}$ & $\begin{array}{c}\text { Mental } \\
\text { disorders }\end{array}$ & $\begin{array}{c}\text { Other } \\
\text { diagnoses }\end{array}$ \\
\hline \multicolumn{8}{|l|}{ Women } \\
\hline$<20$ & 328 & 6 & 2816 & 0.99 & 0.32 & 0.28 & 0.39 \\
\hline $20-24.9$ & 2418 & 47 & 19896 & 0.95 & 0.37 & 0.30 & 0.28 \\
\hline $25-29.9$ & 1638 & 32 & 12215 & 1.60 & 0.78 & 0.40 & 0.43 \\
\hline $30-34.9$ & 570 & 11 & 4219 & 2.28 & 1.07 & 0.57 & 0.64 \\
\hline$\geq 35$ & 177 & 3 & 1244 & 3.86 & 2.09 & 0.80 & 0.96 \\
\hline \multicolumn{8}{|l|}{ Men } \\
\hline$<20$ & 21 & 1 & 168 & . & . & . & . \\
\hline $20-24.9$ & 530 & 38 & 4211 & 0.86 & 0.21 & 0.31 & 0.33 \\
\hline $25-29.9$ & 643 & 46 & 4619 & 1.34 & 0.37 & 0.48 & 0.50 \\
\hline $30-34.9$ & 165 & 12 & 1130 & 1.77 & 0.88 & 0.53 & 0.35 \\
\hline$\geq 35$ & 52 & 4 & 347 & 3.46 & 2.31 & 0.29 & 0.86 \\
\hline
\end{tabular}

Table 2. Description of variables by body mass index (BMI) groups among women and men.

\begin{tabular}{|c|c|c|c|c|c|c|c|c|c|c|c|c|c|c|c|c|}
\hline & \multicolumn{15}{|c|}{$\mathrm{BMI}\left(\mathrm{kg} / \mathrm{m}^{2}\right)$} & \multirow[t]{3}{*}{$P$-value a } \\
\hline & \multicolumn{3}{|c|}{$<20$} & \multicolumn{3}{|c|}{$20-24.9$} & \multicolumn{3}{|c|}{$25-29.9$} & \multicolumn{3}{|c|}{$30-34.9$} & \multicolumn{3}{|c|}{$\geq 35$} & \\
\hline & $\mathrm{N}$ & Mean & $\%$ & $\mathrm{~N}$ & Mean & $\%$ & $\mathrm{~N}$ & Mean & $\%$ & $\mathrm{~N}$ & Mean & $\%$ & $\mathrm{~N}$ & Mean & $\%$ & \\
\hline Women & 328 & & & 2418 & & & 1638 & & & 570 & & & 177 & & & \\
\hline Age (years) & & 47 & & & 49 & & & 50 & & & 50 & & & 51 & & $<0.01$ \\
\hline Diagnosed diseases $(\geq 1)$ & & & 44 & & & 51 & & & 59 & & & 61 & & & 73 & $<0.01$ \\
\hline Physical functioning & & 52 & & & 51 & & & 48 & & & 46 & & & 43 & & $<0.01$ \\
\hline Mental functioning & & 51 & & & 51 & & & 52 & & & 52 & & & 53 & & 0.04 \\
\hline Hazardous exposures (<median) & & & 53 & & & 53 & & & 47 & & & 49 & & & 40 & $<0.01$ \\
\hline Physical workload (<median) & & & 54 & & & 53 & & & 47 & & & 45 & & & 42 & $<0.01$ \\
\hline Computer work (<median) & & & 51 & & & 47 & & & 52 & & & 55 & & & 56 & $<0.01$ \\
\hline Shift work (positive) & & & 17 & & & 19 & & & 23 & & & 25 & & & 27 & $<0.01$ \\
\hline Job demands (<median) & & & 54 & & & 53 & & & 54 & & & 50 & & & 53 & 0.67 \\
\hline Job control (<median) & & & 45 & & & 46 & & & 49 & & & 48 & & & 57 & 0.02 \\
\hline Men & 21 & & & 530 & & & 643 & & & 165 & & & 52 & & & \\
\hline Age (years) & & 50 & & & 49 & & & 50 & & & 52 & & & 51 & & $<0.01$ \\
\hline Diagnosed diseases $(\geq 1)$ & & & 52 & & & 42 & & & 51 & & & 64 & & & 71 & $<0.01$ \\
\hline Physical functioning & & 50 & & & 52 & & & 51 & & & 48 & & & 43 & & $<0.01$ \\
\hline Mental functioning & & 55 & & & 51 & & & 52 & & & 52 & & & 51 & & 0.51 \\
\hline Hazardous exposures (<median) & & & 71 & & & 53 & & & 50 & & & 41 & & & 38 & 0.01 \\
\hline Physical workload (<median) & & & 48 & & & 55 & & & 49 & & & 41 & & & 44 & 0.02 \\
\hline Computer work (<median) & & & 52 & & & 50 & & & 49 & & & 49 & & & 63 & 0.40 \\
\hline Shift work (positive) & & & 14 & & & 26 & & & 32 & & & 33 & & & 27 & 0.10 \\
\hline Job demands (<median) & & & 71 & & & 47 & & & 49 & & & 45 & & & 55 & 0.18 \\
\hline Job control (<median) & & & 52 & & & 46 & & & 46 & & & 59 & & & 48 & 0.03 \\
\hline All & 349 & & & 2948 & & & 2281 & & & 735 & & & 229 & & & \\
\hline Age (years) & & 47 & & & 49 & & & 50 & & & 51 & & & 51 & & $<0.01$ \\
\hline Diagnosed diseases $(\geq 1)$ & & & 45 & & & 49 & & & 57 & & & 61 & & & 72 & $<0.01$ \\
\hline Physical functioning & & 52 & & & 51 & & & 49 & & & 46 & & & 43 & & $<0.01$ \\
\hline Mental functioning & & 51 & & & 51 & & & 52 & & & 52 & & & 52 & & 0.12 \\
\hline Hazardous exposures (<median) & & & 54 & & & 53 & & & 48 & & & 47 & & & 40 & $<0.01$ \\
\hline Physical workload (<median) & & & 54 & & & 53 & & & 48 & & & 44 & & & 43 & $<0.01$ \\
\hline Computer work (<median) & & & 51 & & & 47 & & & 52 & & & 53 & & & 58 & $<0.01$ \\
\hline Shift work (positive) & & & 17 & & & 20 & & & 26 & & & 27 & & & 27 & $<0.01$ \\
\hline Job demands (<median) & & & 55 & & & 52 & & & 53 & & & 49 & & & 53 & 0.39 \\
\hline Job control (<median) & & & 45 & & & 46 & & & 48 & & & 51 & & & 55 & 0.01 \\
\hline
\end{tabular}

a F-test for means, Chi-square test for proportions. 
Table 3. The hazard ratios (HR) and their $95 \%$ confidence intervals $(95 \% \mathrm{Cl})$ for all cause-disability retirement among women and men between body mass index (BMI) groups, P-value for overall differences between BMI groups.

\begin{tabular}{|c|c|c|c|c|c|c|c|c|c|c|c|}
\hline & \multicolumn{10}{|c|}{$\mathrm{BMI}\left(\mathrm{kg} / \mathrm{m}^{2}\right)$} & \multirow[t]{3}{*}{ P-value } \\
\hline & \multicolumn{2}{|c|}{$<20$} & \multicolumn{2}{|c|}{$20-24.9$} & \multicolumn{2}{|c|}{$25-29.9$} & \multicolumn{2}{|c|}{$30-34.9$} & \multicolumn{2}{|c|}{$>35$} & \\
\hline & $\mathrm{HR}$ & $95 \% \mathrm{Cl}$ & $\mathrm{HR}$ & $95 \% \mathrm{Cl}$ & $\mathrm{HR}$ & $95 \% \mathrm{Cl}$ & $\mathrm{HR}$ & $95 \% \mathrm{Cl}$ & $\mathrm{HR}$ & $95 \% \mathrm{Cl}$ & \\
\hline \multicolumn{12}{|l|}{ Women } \\
\hline Model 1 a & 1.30 & $0.82-1.93$ & 1 & .. & 1.38 & $1.11-1.72$ & 2.05 & $1.58-2.67$ & 3.47 & $2.47-4.87$ & $<0.01$ \\
\hline Model 2 b & 1.28 & $0.84-1.96$ & 1 & .. & 1.24 & $0.99-1.54$ & 1.76 & $1.35-2.29$ & 2.56 & $1.80-3.63$ & $<0.01$ \\
\hline Model $3^{c}$ & 1.27 & $0.83-1.96$ & 1 & .. & 1.10 & $0.88-1.37$ & 1.38 & $1.06-1.80$ & 2.12 & $1.50-2.99$ & $<0.01$ \\
\hline Model $4^{d}$ & 1.23 & $0.82-1.92$ & 1 & .. & 1.23 & $1.00-1.53$ & 1.89 & $1.45-2.46$ & 2.94 & $2.09-4.14$ & $<0.01$ \\
\hline Model $5^{\mathrm{e}}$ & 1.29 & $0.84-1.99$ & 1 & .. & 1.02 & $0.82-1.27$ & 1.33 & $1.02-1.74$ & 1.73 & $1.20-2.49$ & 0.01 \\
\hline \multicolumn{12}{|l|}{ Men } \\
\hline Model $1^{\mathrm{a}}$ & & & 1 & .. & 1.49 & $0.96-2.32$ & 1.45 & $0.78-2.69$ & 3.32 & $1.58-6.98$ & 0.03 \\
\hline Model 2 b & & & 1 & .. & 1.42 & $0.90-2.22$ & 1.02 & $0.54-1.95$ & 1.56 & $0.70-3.49$ & 0.51 \\
\hline Model $3^{c}$ & & & 1 & .. & 1.43 & $0.92-2.23$ & 1.16 & $0.62-2.18$ & 2.11 & $0.99-4.50$ & 0.30 \\
\hline Model $4^{d}$ & & & 1 & .. & 1.51 & $0.97-2.37$ & 1.35 & $0.72-2.51$ & 3.04 & $1.43-6.45$ & 0.05 \\
\hline Model $5^{\mathrm{e}}$ & & & 1 & .. & 1.45 & $0.92-2.30$ & 0.95 & $0.49-1.84$ & 1.19 & $0.51-2.78$ & 0.45 \\
\hline
\end{tabular}

adjusted for age.

${ }^{\mathrm{b}}$ Adjusted for age and diagnosed diseases (cardiovascular diseases, musculoskeletal diseases, mental disorders, diabetes, eating disorders, cancer).

${ }^{c}$ Adjusted for age and physical and mental functioning.

adjusted for age and working conditions (shift work, physical working conditions, psychosocial stress at work).

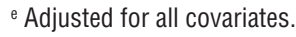

severely obese the elevated risk remained. Sensitivity analysis showed that when physical and mental functioning were adjusted for in separate models, physical functioning attenuated the association further (data not shown). Adjusting for working conditions also slightly attenuated the association between BMI and disability retirement. When adjusting for all covariates simultaneously, the risk for disability retirement remained elevated among the severely obese (HR $1.73,95 \%$ CI 1.20-2.49) and the obese (HR 1.33, 95\% CI 1.02-1.74).

There were no disability retirement events among underweight men. Otherwise the association between BMI and all-cause retirement was similar to that for women, but the elevated risk for all-cause disability retirement was statistically significant only among the severely obese (HR 3.32, 95\% CI 1.58-6.98) when adjusting for age. Adjusting for diseases and physical and mental functioning, the association lost statistical significance.

\section{Diagnosis-specific retirement}

When pooling the data of men and women together, severely obese (HR 3.45, 95\% CI 2.53-4.69), obese (HR $1.94,95 \%$ CI 1.52-2.46) and overweight (HR 1.42, 95\% CI 1.17-1.72) participants had a higher risk of disability retirement after adjusting for age and gender (table 4). Being underweight was not statistically significantly associated with disability retirement. Adjusting for all covariates attenuated the association clearly, but the risk for all-cause disability retirement was still elevated among the severely obese (HR 1.68, 95\% CI 1.22-2.35) and obese (HR 1.27, 95\% CI 1.00-1.63). Of the dis- ability retirements, $43 \%$ were due to musculoskeletal diseases, $29 \%$ were due to other causes, and $28 \%$ were due to mental disorders.

The age- and gender-adjusted association of BMI with disability retirement due to musculoskeletal diseases was stronger than for all-cause disability retirement when adjusting for age and gender (table 4). The risk was not elevated among the underweight. When adjusting for diseases, BMI remained strongly associated with disability retirement due to musculoskeletal diseases. When adjusting for physical and mental functioning, the risk for disability retirement due to musculoskeletal diseases attenuated markedly and only the severely obese had an elevated risk for disability retirement. Adjusting for working conditions slightly attenuated the risk among the severely obese and, to a lesser extent, also the obese and overweight. After adjusting for all covariates, only the severely obese had an elevated risk for musculoskeletal disability retirement.

Disability retirement due to mental disorders differed from that due to all causes as the association was weaker. When adjusting for age and gender, the severely obese and obese had elevated risk of disability retirement. When adjusting for diseases, only the severely obese retained the elevated risk. Adjustments for working conditions attenuated the risk for disability retirement only slightly. In the fully adjusted model, BMI was no longer associated with the risk for disability retirement.

The severely obese and the obese also had an elevated risk for disability retirement due to other causes. Although the underweight did not have a statistically significantly increased risk, the risk estimate was higher than for retirement due to musculoskeletal diseases or 
Table 4. The hazard ratios (HR) and their $95 \%$ confidence intervals $(95 \% \mathrm{Cl})$ for a disability retirement due to all cause, musculoskeletal disorders, psychiatric disorders, and other conditions among both women and men between BMI groups; P-value for overall differences between BMI-groups.

\begin{tabular}{|c|c|c|c|c|c|c|c|c|c|c|c|}
\hline & \multicolumn{8}{|c|}{$\mathrm{BMI}\left(\mathrm{kg} / \mathrm{m}^{2}\right)$} & & & \multirow[t]{3}{*}{ P-value } \\
\hline & \multicolumn{2}{|c|}{$<20$} & \multicolumn{2}{|c|}{$20-24.9$} & \multicolumn{2}{|c|}{$25-29.9$} & \multicolumn{2}{|c|}{$30-34.5$} & \multicolumn{2}{|c|}{$\geq 35$} & \\
\hline & $\mathrm{HR}$ & $95 \% \mathrm{Cl}$ & $\mathrm{HR}$ & $95 \% \mathrm{Cl}$ & $\mathrm{HR}$ & $95 \% \mathrm{Cl}$ & $\mathrm{HR}$ & $95 \% \mathrm{Cl}$ & $\mathrm{HR}$ & $95 \% \mathrm{Cl}$ & \\
\hline \multicolumn{12}{|l|}{ All cause } \\
\hline Model 1 a & 1.16 & $0.76-1.77$ & 1 & .. & 1.42 & $1.17-1.72$ & 1.94 & $1.52-2.46$ & 3.45 & $2.53-4.69$ & $<0.01$ \\
\hline Model $2^{b}$ & 1.15 & $0.75-1.76$ & 1 & .. & 1.29 & $1.05-1.55$ & 1.61 & $1.26-2.05$ & 2.39 & $1.73-3.30$ & $<0.01$ \\
\hline Model $3^{c}$ & 1.17 & $0.77-1.79$ & 1 &.. & 1.17 & $0.97-1.43$ & 1.34 & $1.05-1.72$ & 2.14 & $1.56-2.92$ & $<0.01$ \\
\hline Model 4 d & 1.19 & $0.78-1.82$ & 1 &.. & 1.29 & $1.06-1.57$ & 1.77 & $1.39-2.25$ & 2.93 & $2.15-4.00$ & $<0.01$ \\
\hline Model 5 e & 1.19 & $0.78-1.83$ & 1 & .. & 1.10 & $0.90-1.34$ & 1.27 & $1.00-1.63$ & 1.68 & $1.22-2.35$ & 0.02 \\
\hline \multicolumn{12}{|c|}{ Musculoskeletal } \\
\hline Model 1 a & 1.18 & $0.59-2.37$ & 1 & .. & 1.69 & $1.25-2.30$ & 2.35 & $1.62-3.39$ & 4.76 & $3.06-7.40$ & $<0.01$ \\
\hline Model 2 b & 1.20 & $0.60-2.40$ & 1 &.. & 1.49 & $1.10-2.03$ & 1.89 & $1.30-2.75$ & 3.14 & $1.98-4.96$ & $<0.01$ \\
\hline Model $3^{c}$ & 1.25 & $0.63-2.50$ & 1 &.. & 1.27 & $0.94-1.73$ & 1.38 & $0.95-2.00$ & 2.34 & $1.49-3.66$ & 0.01 \\
\hline Model $4^{d}$ & 1.20 & $0.60-2.41$ & 1 &.. & 1.46 & $1.08-1.98$ & 2.02 & $1.40-2.93$ & 3.63 & $2.32-5.68$ & $<0.01$ \\
\hline Model 5 e & 1.33 & $0.66-2.67$ & 1 & .. & 1.19 & $0.87-1.62$ & 1.35 & $0.92-1.96$ & 1.79 & $1.12-2.87$ & 0.14 \\
\hline \multicolumn{12}{|l|}{ Psychiatric } \\
\hline Model1 a & 0.89 & $0.39-2.07$ & 1 & .. & 1.11 & $0.77-1.61$ & 1.61 & $1.02-2.54$ & 2.40 & $1.26-4.56$ & 0.03 \\
\hline Model $2^{b}$ & 0.90 & $0.39-2.08$ & 1 &.. & 1.04 & $0.72-1.50$ & 1.30 & $0.82-2.08$ & 1.96 & $1.01-3.80$ & 0.27 \\
\hline Model $3^{c}$ & 0.88 & $0.38-2.04$ & 1 &.. & 0.99 & $0.69-1.44$ & 1.27 & $0.79-2.02$ & 1.79 & $0.93-3.44$ & 0.37 \\
\hline Model 4 d & 0.95 & $0.41-2.20$ & 1 &.. & 1.08 & $0.75-1.56$ & 1.54 & $0.97-2.45$ & 2.17 & $1.14-4.12$ & 0.07 \\
\hline Model 5 e & 0.88 & $0.38-2.06$ & 1 &.. & 0.96 & $0.66-1.39$ & 1.16 & $0.72-1.87$ & 1.63 & $0.83-3.21$ & 0.56 \\
\hline \multicolumn{12}{|c|}{ Other causes } \\
\hline Model 1 a & 1.40 & $0.70-2.83$ & 1 & .. & 1.38 & $0.97-1.96$ & 1.76 & $1.12-2.76$ & 2.88 & $1.58-5.25$ & 0.01 \\
\hline Model $2^{b}$ & 1.38 & $0.68-2.79$ & 1 & .. & 1.26 & $0.89-1.80$ & 1.45 & $0.92-2.30$ & 1.97 & $1.05-3.71$ & 0.22 \\
\hline Model $3^{c}$ & 1.42 & $0.70-2.87$ & 1 & .. & 1.22 & $0.86-1.75$ & 1.35 & $0.86-2.14$ & 2.04 & $1.11-3.77$ & 0.21 \\
\hline Model $4^{d}$ & 1.43 & $0.71-2.89$ & 1 & .. & 1.29 & $0.90-1.83$ & 1.67 & $1.06-2.61$ & 2.70 & $1.48-4.94$ & 0.01 \\
\hline Model 5 e & 1.41 & $0.70-2.85$ & 1 & .. & 1.15 & $0.80-1.65$ & 1.21 & $0.76-1.93$ & 1.65 & $0.87-3.14$ & 0.64 \\
\hline
\end{tabular}

adjusted for age and gender.

${ }^{\mathrm{b}}$ Adjusted for age, gender, and diagnosed diseases (cardiovascular diseases, musculoskeletal diseases, mental disorders, diabetes, eating disorders, cancer).

c Adjusted for age, gender, and physical and mental functioning.

d Adjusted for age, gender, and working conditions (shift work, physical working conditions, psychosocial stress at work).

e Adjusted for all covariates.

mental disorders. When adjusting for diseases and physical and mental functioning, the elevated risk among the severely obese remained. Again, adjusting for working conditions had negligible effects on the association. When all covariates were simultaneously adjusted for, no statistically significant association between BMI and disability retirement due to other causes remained.

\section{Discussion}

This study sought to examine the associations between relative weight and disability retirement. There were three main findings. First, BMI was strongly associated with all-cause disability retirement both among women and men. The risk of disability retirement was particularly clear among the obese and the severely obese, but even the overweight had an elevated risk for all-cause disability retirement. However, being underweight was not associated with disability retirement. Second, BMI was strongly associated with disability retirement due to musculoskeletal diseases and, to a lesser extent, disability retirement due to mental disorders and other causes. Third, adjusting for diseases and physical and mental functioning at baseline attenuated the associations clearly; however working conditions had negligible effects on the associations.

Similar results have been reported previously (12, 13, 23). In particular, our age-adjusted results concur with those of an earlier Finnish study using retirement data from the 1980s (12). Our study extends these previous results as the BMI grouping used in our study enabled more detailed analyses especially among the severely obese, and the range of adjustments was wider, giving a more comprehensive picture of the associations between relative weight and disability retirement.

The risk for disability retirement for men and women appeared to be approximately on the same level among the severely obese and the overweight, although statistical significance was not reached among overweight men. This is in accordance with earlier studies among men, where being overweight was marginally associated with the risk of disability retirement $(13,23)$. In our study, 
obese men tended to have a somewhat lower risk for disability retirement than obese women.

While previous studies suggest that BMI has a J-shaped association with disability retirement (11), the risk among the underweight was not statistically significantly increased in our study. However, the risk estimates among underweight women were consistently elevated and adjustments did not change the estimates notably. Although this result is tentative, it is possible that being underweight is associated with other health problems thus leading to increased risk of disability retirement.

When considering disability retirement according to diagnosis, disability retirements due to musculoskeletal disorders had the strongest association with BMI. The risk was noticeable among the severely obese, obese, and overweight. This is in accordance with previous studies (12-14). However, the age-adjusted risk estimate for the severely obese was considerably higher in our study than earlier ones, possibly because earlier studies did not separate the severely obese from the obese. It is well known that obesity is associated with the development of several musculoskeletal diseases such as knee and hip arthrosis and back pain (24). Obesity's effect on the pathogenesis of these diseases is still unclear, but in addition to functional and structural strain of the musculoskeletal system, also inflammatory processes in the excess adipose tissue may contribute to the pathogenesis (24).

In previous research, obesity and mental disorders have been reciprocally linked (25). The elevated risk for disability retirement due to mental disorders appeared among the severely obese and the obese. However, when adjusting for physical and mental functioning, the association attenuated and lost statistical significance. This is reasonable since, in order for a mental disorder to cause permanent work disability, it has to lower employees functioning in many areas of life (26). Without a major impairment in functioning, there is no basis for a disability retirement. The older Finnish study (12) did not detect an association between BMI and disability retirement due to mental disorders. Other previous studies with information on diagnoses leading to disability retirement $(13,14)$ only analyzed men. In those studies relative weight was associated with a risk of disability retirement due to mental disorders. Again, our study had a more comprehensive range of covariate adjustments than these earlier studies, which were based on sociodemographic covariates, such as age and socioeconomic position. Covariates such as working conditions, previously diagnosed diseases, and mental and physical functioning have not been previously considered to the same extent.

The severely obese and the obese also had an increased risk of disability retirement due to other causes. The most common diagnosis group in this category was malignant neoplasms (ICD- 10 codes $\mathrm{C} 00$ C97), followed by diseases of the nervous system (ICD10 codes G00-G99), and diseases of the circulatory system (ICD-10 codes I00-I99) (data not shown). As obesity is associated with many cancers and cardiovascular diseases (1), the result is reasonable. The diseases of the nervous system were heterogeneous in our study population, but among those diseases multiple sclerosis was the most common diagnosis for disability retirement. Interestingly, obesity during adolescence though not adulthood $(27,28)$ has been associated with the development of multiple sclerosis in later life. Among the underweight, the risk for disability retirement due to other causes tended to be higher than for musculoskeletal diseases and mental disorders, although this finding was not statistically significant. This could be the result of underlying undiagnosed health problems, such as neurologic diseases, malignant neoplasms, or alcohol problems leading to weight loss.

When the possibility of granting a disability pension is assessed, an individual's work ability is evaluated with respect to health, functioning, and working conditions; mere obesity is not a basis for disability retirement. We therefore took into account these three factors that may contribute to the association between relative weight and disability retirement. Working conditions had only slight effects on the association between relative weight and disability retirement, whereas diseases attenuated the risk somewhat among the severely obese, obese, and overweight, and physical and mental functioning had the strongest effect. This could imply that the association of obesity with disability retirement is to a large part affected by the loss of functioning, especially physical functioning. This was especially true when considering disability retirement due to musculoskeletal diseases among the severely obese and obese. The physical component summary consists of various factors, the main areas being physical functioning, role limitations due to physical problems, bodily pain, and general health perceptions. In addition to promoting the maintenance of normal weight, it could be feasible to focus on functioning in relation to obesity in order to prevent the adverse consequences of obesity to work disability.

\section{Methodological considerations}

The main strengths of this study include reliable register-based data on disability retirements and the prospective study design. In addition, the data on disability retirement also included the main causes of disability. The comprehensive baseline survey data comprising a large number of participants enabled the study to take into account a range of key covariates. The data on weight and height were self-reported, and although the 
use of self-reported values has been found to be appropriate in epidemiological studies $(29,30)$, this may lead to overestimation of the risk especially in the higher BMI groups. Overweight and obese people are known to underestimate their weight and overestimate their height more than normal-weight individuals (31) thus causing possible misplacement in the lower BMI category.

Although working conditions, health status, and functioning were based on self-reports, the information was gathered using validated and reliable instruments. However, we lack information on the length of exposure to the given conditions. For example, baseline working conditions might have changed during follow-up or even earlier preventing or postponing future disability retirement. This might lead to an underestimation of their role in our study. Finally, the Helsinki Health Study cohort includes a broad variety of white- and blue-collar occupations (17) from a large municipal employer. The results are likely to reflect the municipal sector and possibly also the public sector more generally. However, generalizations to the work force more broadly are not warranted.

\section{Concluding remarks}

In this study, the severely obese and obese had a clearly elevated risk for disability retirement, especially for musculoskeletal causes. The adverse effects of obesity on health and work ability are partly dependent on comorbidities and ensuing lowered physical functioning. Our results emphasize the importance of promoting the maintenance of normal weight among employees. In addition, efforts should be made to improve physical functioning of the obese via rehabilitation and effectively treat obesity and its co-morbidities to counteract the elevated risk of disability retirement. Occupational healthcare plays a key role as assessing and preventing the loss of work ability are among the major challenges to occupational healthcare.

\section{Acknowledgments}

The Academy of Finland supported this study. The National Graduate School of Clinical Investigation supported Eira Roos. We thank the City of Helsinki for permission to use the data and all participating employees. The authors declare no conflicts of interest.

\section{References}

1. Guh DP, Zhang W, Bansback N, Amarsi Z, Birmingham CL, Anis AH. The incidence of co-morbidities related to obesity and overweight: A systematic review and meta-analysis. BMC Public Health. 2009:1-20.

2. McGee DL, Diverse Populations Collaboration. Body mass index and mortality: A meta-analysis based on person-level data from twenty-six observational studies. Ann Epidemiol. 2005 Feb;15(2):87-97. http://dx.doi.org/10.1016/j. annepidem.2004.05.012.

3. Doll HA, Petersen SE, Stewart-Brown SL. Obesity and physical and emotional well-being: Associations between body mass index, chronic illness, and the physical and mental components of the SF-36 questionnaire. Obes Res. 2000 Mar;8(2):160-70. http://dx.doi.org/10.1038/oby.2000.17.

4. Soltoft F, Hammer M, Kragh N. The association of body mass index and health-related quality of life in the general population: Data from the 2003 health survey of england. Qual Life Res. 2009 Dec;18(10):1293-9. http://dx.doi. org/10.1007/s11136-009-9541-8.

5. Neovius K, Johansson K, Kark M, Neovius M. Obesity status and sick leave: A systematic review. Obes Rev. 2009 Jan;10(1):17-27. http://dx.doi.org/10.1111/j.1467789X.2008.00521.x.

6. van Duijvenbode DC, Hoozemans MJ, van Poppel MN, Proper KI. The relationship between overweight and obesity, and sick leave: A systematic review. Int J Obes (Lond). 2009 Aug;33(8):807-16. http://dx.doi.org/10.1038/ijo.2009.121.

7. Schmier JK, Jones ML, Halpern MT. Cost of obesity in the workplace. Scand J Work Environ Health. 2006 Feb;32(1):511. http://dx.doi.org/10.5271/sjweh.970.

8. Ricci JA, Chee E. Lost productive time associated with excess weight in the U.S. workforce. J Occup Environ Med. 2005 Dec;47(12):1227-34. http://dx.doi.org/10.1097/01. jom.0000184871.20901.c3.

9. Wolfenstetter SB. Future direct and indirect costs of obesity and the influence of gaining weight: Results from the MONICA/KORA cohort studies, 1995-2005. Econ Hum Biol. Mar;10(2):127-38.

10. Renna F, Thakur N. Direct and indirect effects of obesity on U.S. labor market outcomes of older working age adults. Soc Sci Med. 2010;71(2):405-13. http://dx.doi.org/10.1016/j. socscimed.2010.03.038.

11. Neovius K, Johansson K, Rossner S, Neovius M. Disability pension, employment and obesity status: A systematic review. Obes Rev. 2008 Nov;9(6):572-81. http://dx.doi.org/10.1111/ j.1467-789X.2008.00502.x.

12. Rissanen A, Heliovaara M, Knekt P, Reunanen A, Aromaa A, Maatela J. Risk of disability and mortality due to overweight in a Finnish population. BMJ. 1990 Oct 13;301(6756):835-7. http://dx.doi.org/10.1136/bmj.301.6756.835.

13. Mansson NO, Eriksson KF, Israelsson B, Ranstam J, Melander A, Rastam L. Body mass index and disability pension in 
middle-aged men--non-linear relations. Int J Epidemiol. 1996 Feb;25(1):80-5. http://dx.doi.org/10.1093/ije/25.1.80.

14. Neovius M, Kark M, Rasmussen F. Association between obesity status in young adulthood and disability pension. Int $\mathrm{J}$ Obes. 2008 08;32(8):1319-26.

15. Lahelma E, Laaksonen M, Lallukka T, Martikainen P, Pietiläinen O, Saastamoinen P, et al. Working conditions as risk factors for disability retirement: A longitudinal register linkage study. BMC Public Health. 2012;12:309. http://dx.doi. org/10.1186/1471-2458-12-309.

16. Friis K, Ekholm O, Hundrup YA. The relationship between lifestyle, working environment, socio-demographic factors and expulsion from the labour market due to disability pension among nurses. Scand J Caring Sci. 2008;22(2):241-8. http:// dx.doi.org/10.1111/j.1471-6712.2007.00521.x.

17. Lahelma E, Aittomäki A, Laaksonen M, Lallukka T, Martikainen P, Piha K, et al. Cohort profile: The Helsinki Health Study. Int J Epidemiol. 2012 Mar 31. [Epub ahead of print] http://dx.doi.org/10.1093/ije/dys039.

18. Laaksonen M, Aittomaki A, Lallukka T, Rahkonen O, Saastamoinen P, Silventoinen K, et al. Register-based study among employees showed small nonparticipation bias in health surveys and check-ups. J Clin Epidemiol. 2008 Sep;61(9):9006. http://dx.doi.org/10.1016/j.jclinepi.2007.09.010.

19. World Health Organization. International statistical classification of diseases and related health problems, the ICD10, volume 1. 2nd ed, 10th revision ed. Geneva: World Health Organization; 2004.

20. Ware JE, Snow KK, Kosisnki M, Gandek B. SF-36 Health Survey Manual and Interpretation Guide. New England Medical Center Hospital. Boston: The Health Institute, New England Medical Center; 1993.

21. Piirainen H, Hirvonen M, Elo A-L, Huuhtanen P, Kandolin I, Kauppinen K, et al. The work and health interview study 2003. basic report. Helsinki, Finland: Finnish Institute of Occupational Health; 2003.

22. Karasek R. Job content questionnaire and user's guide. Lowell, MA, USA: Department of Work Environment, University of Massachusetts; 1985.
23. Karnehed N, Rasmussen F, Kark M. Obesity in young adulthood and later disability pension: A population-based cohort study of 366,929 Swedish men. Scand J Public Health. 2007;35(1):48 54. http://dx.doi.org/10.1080/14034940600858524.

24. Anandacoomarasamy A. Obesity and the musculoskeletal system. Curr Opin Rheumatol. 2009;21(1):71. http://dx.doi. org/10.1097/BOR.0b013e32831bc0d7.

25. Luppino FS, de Wit LM, Bouvy PF, Stijnen T, Cuijpers P, Penninx BV, et al. Overweight, obesity, and depression: A systematic review and meta-analysis of longitudinal studies. Arch Gen Psychiatry. 2010 March;67(3):220-9. http://dx.doi. org/10.1001/archgenpsychiatry.2010.2.

26. Act 549/2003 [kunnallinen eläkelaki (Act on communal pension), 3. chapter, § 24] [Internet]. Available from: http:// www.finlex.fi/fi/laki/alkup/2003/20030549.

27. Hedström A.K. High body mass index before age 20 is associated with increased risk for multiple sclerosis in both men and women. Mult Scler. 2012 Sep;18(9):1334-6. http:// dx.doi.org/10.1177/1352458512436596.

28. Munger KL. Body size and risk of MS in two cohorts of US women. Neurology. 2009;73(19):1543-50. http://dx.doi. org/10.1212/WNL.0b013e3181c0d6e0.

29. McAdams MA, Van Dam RM, Hu FB. Comparison of selfreported and measured BMI as correlates of disease markers in US adults. Obesity (Silver Spring). 2007 Jan;15(1):188-96. http://dx.doi.org/10.1038/oby.2007.504

30. Stommel M, Schoenborn CA. Accuracy and usefulness of BMI measures based on self-reported weight and height: Findings from the NHANES \& NHIS 2001-2006. BMC Public Health. 2009 Nov 19;9:421. http://dx.doi. org/10.1186/1471-2458-9-421.

31. Shields M, Gorber SC, Tremblay MS. Estimates of obesity based on self-report versus direct measures. Health reports / Statistics Canada, Canadian Centre for Health Information. Centre Canadien d'Information sur la Santé. 2008;19(2):6176. http://ukpmc.ac.uk/abstract/MED/18642520/reload $=0 ;$ j sessionid=9yOm18aZjOVS8rO30HVM.0.

Received for publication: 19 June 2012 